\title{
Gastrostomía descompresiva en obstrucción gastrointestinal maligna: reporte de caso y revisión de la literatura
}

\section{Decompressive gastrostomy in patients with malignant gastrointestinal obstruction. Case report and literature review}

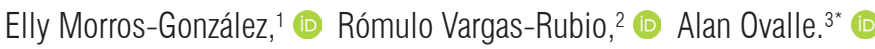

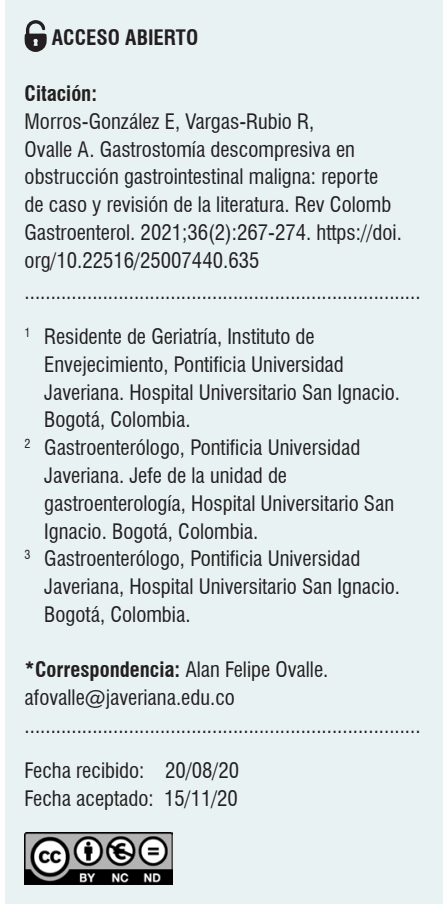

\begin{abstract}
Resumen
La gastrostomía descompresiva durante los últimos años ha surgido como una alternativa de manejo para la paliación de síntomas en personas con enfermedad oncológica terminal que desarrollan obstrucción intestinal sin indicación quirúrgica inicial y es refractaria al manejo médico. El objetivo es brindar calidad de vida a través de la restauración de la vía oral. Sus contraindicaciones han variado con el tiempo; sin embargo, han surgido nuevas técnicas que permiten ampliar el espectro de indicaciones de este procedimiento. Se ha descrito que permite apoyar el control de síntomas, el retorno de la persona a su lugar de cuidado y, posiblemente, el reinicio de la vía oral con la intención de mantener la calidad de vida de la persona.
\end{abstract}

\section{Palabras clave}

Neoplasia, obstrucción gastrointestinal maligna, gastrostomía descompresiva.

\section{Abstract}

In recent years, decompressive gastrostomy has emerged as a therapeutic option for people with terminal cancer who experience intestinal obstruction without an initial surgical indication and refractory to medical treatment. The objective is to provide a better quality of life by restoring the oral route. Its contraindications have varied over time; however, new techniques have allowed broadening the spectrum of indications for this procedure. It has been reported that this technique supports symptom control and allows the return of the patients to their place of care. It could also allow restarting the oral route in order to maintain quality of life.

\section{Keywords}

Neoplasm; Malignant bowel obstruction; Decompressive gastrostomy.

\section{INTRODUCCIÓN}

La presencia de la obstrucción gastrointestinal maligna (OGIM) condiciona un importante deterioro de la calidad de vida y es de mal pronóstico por la alta mortalidad a corto plazo, alta recurrencia y refractariedad al manejo médico paliativo. Adicionalmente, el gusto y la posibilidad de ingerir alimentos se ve limitada y esto puede influir seriamente en la calidad de vida de las personas con enfermedad neoplásica avanzada.
La prevalencia de OGIM puede alcanzar entre el $3 \%$ y el $15 \%$ de las personas con cáncer avanzado (1-3). Hasta dos tercios de los casos se originan de tumores en el intestino delgado; un tercio de los casos, de tumores en el intestino grueso; y el $20 \%$, en ambos. La presencia de síntomas estará determinada por el nivel de obstrucción, y las náuseas, el vómito, el dolor y la distensión abdominal son los principales. Por una parte, las neoplasias primarias que más frecuentemente causan OGIM son principalmente cáncer de ovario (20\%-50\%), colorrectal (10\%-29\%), gástrico 
(6 \%-19\%), pancreático (6\%-13\%), de vejiga (3\%-10\%), endometrial (3\%-11\%), de mama (2\%-3\%) y melanoma $(3 \%)(4,5)$. A su vez, en el $80 \%$ de los pacientes con cáncer avanzado, la OGIM puede presentar múltiples niveles de obstrucción en relación con un diagnóstico previo de carcinomatosis peritoneal. Por otra parte, puede ser secundaria a su tratamiento, como sucede en la formación de fibrosis o enteritis por radiación, etiología benigna como bridas hasta en un $20 \%$, e indeterminada en un $12 \%(6-8)$.

Hasta un $30 \%$ a $40 \%$ de los pacientes con OGIM inoperable logran la resolución espontánea del episodio obstructivo (8) y se ha estimado una expectativa de vida entre 1 a 9 meses en pacientes con antecedente de cáncer intraabdominal (9, 10). Chakraborty y colaboradores describieron la historia natural de personas con OGIM manejados quirúrgicamente, con quimioterapia o manejo médico únicamente. Aquellos manejados únicamente con medicamentos presentaban un puntaje de 3 y 4 en la escala de la Eastern Cooperative Oncology Group (ECOG), mientras que aquellos intervenidos quirúrgicamente tenían puntaje ECOG de 0 a 2. Los pacientes que recibieron el manejo quirúrgico presentaron menos estancia hospitalaria en comparación con aquellos no manejados quirúrgicamente ( 15 frente a 27 días) (11).

Se ha descrito que el deterioro cognitivo, la caquexia, la disnea en reposo, los tumores abdominales palpables, la insuficiencia hepática, la obstrucción intestinal alta y la deshidratación son factores asociados con la no resolución de la OGIM (8).

El tratamiento está determinado por una evaluación individualizada interdisciplinaria, el estadio de la enfermedad, el pronóstico global y la funcionalidad del paciente, por lo que es fundamental establecer la meta del tratamiento y las preferencias del paciente. Generalmente, el manejo de la OGIM incluye el ajuste del manejo farmacológico paliativo o manejo quirúrgico, teniendo en cuenta la evaluación de factores de mal pronóstico quirúrgico que pudieran traer mayor morbilidad en el paciente y que contraindican la intervención invasiva. En pacientes con carcinomatosis peritoneal y OGIM, la descompresión gastrointestinal con sonda nasogástrica (SNG) sigue siendo la primera línea de manejo; sin embargo, el uso prolongado se asocia con estrés psicológico y potenciales complicaciones como necrosis del ala nasal, desórdenes laríngeos, lesiones esofagogástricas, otitis media y neumonía aspirativa. En general, los pacientes reportan malestar con una SNG a permanencia y puede generar dificultades en su manejo en ambientes no hospitalarios.

En caso de obstrucción maligna refractaria con náuseas y vómito intratable, se podría considerar la gastrostomía endoscópica descompresiva (GED). Según la localización de la obstrucción (proximal o distal) y según el grado de obstrucción (parcial o completa), será la presentación clí- nica de la obstrucción intestinal maligna. En la obstrucción intestinal proximal, el dolor abdominal es abrupto y las náuseas y el vómito son de altos volúmenes; es poco común la distención abdominal, a diferencia de la obstrucción intestinal distal, en la que los síntomas son más insidiosos, y son frecuentes la distención abdominal y el vómito de características fecaloides.

Malone y colaboradores en 1986 reportaron el primer caso de gastrostomía percutánea por radiología como manejo descompresivo; posteriormente, la técnica fue adoptada y modificada por Stellato y Gauderer, quienes describieron los primeros 8 casos de GED percutánea en 1987 (12), en los que se logró eliminar la necesidad de uso de SNG en todos los pacientes y se mantuvo su eficacia por meses y hasta años dentro de este subgrupo de pacientes. La GED es una herramienta que puede ser complementaria al manejo farmacológico de la OGIM, es costo-efectiva y reduce la morbimortalidad. Además, permite el retiro de la SNG y la posibilidad de ingerir alimentos líquidos y cremosos por vía oral, de modo que se preserva el deseo y gusto por los alimentos y se genera la posibilidad del retorno del paciente a su lugar de cuidado.

\section{CASO CLÍNICO}

Se trata de una paciente femenina de 49 años de edad con antecedente de carcinoma seroso micropapilar de ovario sugestivo de tumor de Krukenberg, asociado con carcinomatosis peritoneal irresecable y compromiso a nivel del intestino delgado y grueso, quien fue manejada con segunda línea de quimioterapia (carboxiplatino y doxorrubicina liposomal) y completó 8 ciclos con intención paliativa. Ingresó a urgencias de nuestra institución por múltiples episodios eméticos asociados con distensión abdominal y ausencia de deposiciones, por lo cual se sospechó una obstrucción intestinal maligna y, en principio, se indicó la colocación SNG; de este modo se obtuvo abundante drenaje bilioso y, posteriormente, se realizó una tomografía de abdomen contrastada, en la que se corroboró la presencia de áreas de compresión intestinal secundarias al cuadro conocido de carcinomatosis peritoneal. La paciente continuaba con abundante drenaje por SNG, por lo cual fue valorada por el servicio de cuidados paliativos, quienes consideraron el manejo asociado con ondansetrón, dexametasona y haloperidol, pero la sintomatología persistió a pesar del manejo previamente descrito. Por lo anterior, fue valorada por el servicio de gastroenterología para considerar realización de GED.

Al día siguiente de la valoración por gastroenterología, la paciente presentó un cuadro de hemorragia de vías digestivas altas y anemización significativa, por lo que se realizó una esofagogastroduodenoscopia en la que se documentó 
esofagitis por retención, hernia hiatal de 4 centímetros con erosiones en el saco herniario, pangastritis erosiva y bulboduodenitis erosiva moderada, sin presencia de trasluminación, por lo que se decidió realizar una GED por parte de radiología intervencionista. La paciente presentó durante su estancia hospitalaria un cuadro de sepsis de origen pulmonar, que requirió el aporte de oxígeno suplementario a alto flujo y manejo antimicrobiano. Posteriormente, la paciente manifestó no desear intervenciones invasivas adicionales, y fue valorada por servicio de ética clínica, quienes respetaron el principio de autonomía de la paciente, por lo que se decidió reorientar el esfuerzo terapéutico y continuar el manejo por parte del servicio de cuidados paliativos.

\section{DISCUSIÓN}

La OGIM se define como la presencia de hallazgos en la historia clínica, examen físico o radiológico de obstrucción intestinal, que debe localizarse por debajo del ligamento de Treitz, y está asociado con cáncer primario intrabdominal o cáncer primario no intrabdominal con enfermedad intraperitoneal evidente (13). Usualmente, las personas presentan náuseas, vómito, dolor y distensión abdominal, y ausencia de deposiciones. Aunque el diagnóstico es clínico, la tomografía axial computarizada (TAC) de abdomen contrastado es el estándar de oro y, en caso de que no haya disponibilidad, la radiografía y ecografía de abdomen son otras opciones diagnósticas (7).

Desde el punto de vista fisiopatológico, existen 2 tipos de mecanismos involucrados en la fisiopatología de la OGIM: mecánico y funcional. El primero se encuentra en relación con la compresión del tracto gastrointestinal por una masa tumoral o por metástasis de forma intrínseca o extrínseca; mientras que el segundo se relaciona con la infiltración tumoral de los nervios del plexo mientérico, que genera alteraciones en la motilidad, alteraciones electrolíticas asociadas con la condición de base, o alteraciones derivadas de los efectos secundarios de los medicamentos utilizados. Lo anterior conduce al aumento de secreciones digestivas, $\mathrm{y}$ son de gran volumen, lo que genera un tercer espacio y deteriora el epitelio intestinal; esto promueve la inflamación y alteración de la motilidad (2).

Con respecto al tratamiento, los pilares del manejo de la OGIM consisten en lograr el control del dolor por los síntomas obstructivos y el control de las náuseas y los episodios eméticos y, adicionalmente, considerar una vía de nutrición alterna. Dentro de las medidas iniciales, se incluyó la realización de descompresión con SNG, mantenimiento de la hidratación y alteración electrolítica, reposo intestinal y control del dolor $(7,14)$.

Asimismo, en este punto se debe considerar la indicación quirúrgica de urgencia, evaluando los factores de mal pronóstico quirúrgico. Generalmente, los pacientes con cáncer avanzado no la tienen, pero se podría estar ante un caso de una obstrucción única que podría manejarse quirúrgicamente (15).

Los objetivos del tratamiento deben ser individualizados teniendo en cuenta el pronóstico de la enfermedad, comorbilidades, funcionalidad y opciones de tratamiento, lo cual implica establecer claramente las metas del tratamiento y las preferencias del paciente. En un paciente con enfermedad neoplásica avanzada, los objetivos de tratamiento están enfocados a controlar los síntomas, mantener o mejorar la calidad de vida, en busca del retorno al sitio de cuidado de la persona. Además, con la GED se busca reducir la carga de secreciones gastrointestinales generadas por las medidas farmacológicas y la compresión mecánica.

El tratamiento farmacológico es multimodal: incluye analgesia, manejo de náuseas y vómito, manejo de secreciones intestinales, administración de antiinflamatorios (esteroides) e hidratación parenteral. Hay heterogeneidad en los algoritmos de manejo. Los anticolinérgicos y los antieméticos son los medicamentos de primera línea que permiten disminuir la contracción del músculo liso y la secreción ácida (la metoclopramida está contraindicada en obstrucción intestinal maligna completa) (14). Los corticoides confieren un estado antiinflamatorio, disminuyen el dolor y tienen propiedades antieméticas, sin que se haya encontrado una mejoría de la supervivencia al compararse con placebo (16). Estos medicamentos deberán suspenderse gradualmente si no hay resolución de la obstrucción. Como segunda línea se ha usado la octreotida, un análogo de la somatostatina, cuyo mecanismo de acción se basa en el control de la vasoactividad intestinal, que favorece la disminución de las secreciones intestinales intraluminales (14).

En todo paciente con OGIM se debe integrar al equipo de cuidado paliativo para realizar una valoración multidimensional y un manejo integral en esta enfermedad de alto impacto. Dentro de las medidas a definir está la indicación de nutrición suplementaria de acuerdo con las metas del tratamiento en el paciente, las cuales se traducen en optimizar la calidad de vida y evitar medidas fútiles que puedan prolongar el sufrimiento en caso de pacientes en el fin de su vida.

La alimentación no solo es una necesidad fisiológica del ser humano, sino también un evento social, cultural y de satisfacción. En las personas con enfermedad oncológica avanzada que cursan con OGIM y síntomas refractarios al manejo farmacológico, si el estado clínico del paciente y las preferencias del mismo lo permiten, se podría considerar la realización de una GED con el fin de favorecer la paliación de los síntomas y, además, brindar la posibilidad de mantener la vía oral para preservar su calidad de vida y favorecer la satisfacción del gusto por los alimentos $(3,17)$.

La sensación de alimentación restaurada a través de la GED desde el punto de vista ético podría generar conflicto, 
puesto que en primer lugar se deberían preservar los principios universales de no maleficencia y de justicia, dado que se deben evaluar los posibles riesgos y complicaciones luego de la realización de GED; y en cuanto al principio de justicia, el equipo interdisciplinario que está acompañando al paciente debe considerar su estado clínico, la capacidad de tolerar la intervención y el posible beneficio después del procedimiento, siempre de la mano de los principios de autonomía, tomando en cuenta la decisión del paciente, todo esto en el contexto del principio de beneficencia.

\section{GED}

Durante los últimos años, la GED ha surgido como una herramienta complementaria en el manejo de la OGIM cuando el manejo farmacológico no logra controlar los síntomas y, a su vez, se debe considerar el retiro de la SNG por todos los efectos secundarios que puede condicionar (3).

La GED permite disminuir la cantidad de secreciones gastrointestinales y gas intestinal; adicionalmente, permite el retiro de la SNG, dado que es más seguro que el uso crónico de la SNG, lo que además favorece el retorno al lugar de cuidado $(3,18)$.

\section{Técnica y profilaxis antibiótica}

Las guías de la Sociedad de Radiología recomiendan profilaxis antibiótica con 1 gramo de cefazolina si la técnica es el paso del catéter por cavidad oral; sin embargo, la profilaxis es controvertida cuando se realiza por vía transabdominal para evitar la infección periostomal $(3,19)$.

\section{Contraindicaciones}

En la Tabla 1 se describen las contraindicaciones para GED de acuerdo con lo definido por las guías multidisciplinarias de diferentes sociedades $(3,19)$.

Tabla 1. Contraindicaciones de GED (19)

\begin{tabular}{|ll}
\multicolumn{1}{c}{ Absolutas } & \multicolumn{1}{c|}{ Relativas } \\
\hline $\begin{array}{l}\text { Coagulopatía } \\
\text { incorregible }\end{array}$ & $\begin{array}{l}\text { Hemorragia gastrointestinal reciente (úlcera } \\
\text { péptica con vaso grande identificado o várices } \\
\text { esofágicas) }\end{array}$ \\
\hline Peritonitis bacteriana & Várices asociadas con hipertensión portal \\
\hline
\end{tabular}

Tomado de: Itkin M et al. Gastroenterology. 2011;141(2):742-65.

La gastrostomía percutánea puede ser insertada endoscópicamente, o bajo ecografía, fluoroscopia o guiada por TAC. El debate se centra en cual método se debe usar, radiológico o endoscópico. La mayoría de las sondas de gastrostomía se coloca endoscópicamente; en un estudio se reportó que entre el $18 \%$ y el $35 \%$ fueron colocadas bajo fluoroscopia
(20). Otras series han demostrado que el método radiológico es con frecuencia más exitoso $(19,20)$, mientras que otros estudios han reportado una alta incidencia de obstrucción de la sonda y necesidad de reemplazo de esta. Silas y colaboradores revisaron las indicaciones, complicaciones y resultados del método radiológico frente al endoscópico en 370 pacientes y reportaron que ambos son seguros y efectivos. Sin embargo, en esta serie, las complicaciones tempranas como la infección fueron más frecuentes con el método por fluoroscopia ( $23 \%$ frente a $11 \% ; p=0,002$ ) (21). En un estudio prospectivo, aleatorizado y controlado de gastroyeyunostomía radiológica frente a la GED, el promedio de tiempo del procedimiento fue significativamente diferente ( 53 minutos frente a 24 minutos; $p=0,013$ ) (22).

La presencia de carcinomatosis peritoneal, infiltración tumoral, cirugía gástrica previa e interposición colónica o hepática entre el estómago y la pared abdominal anterior, aunque dificultan la realización de la técnica usual, no se consideran contraindicaciones, pero pueden ser condiciones para la realización de una técnica de gastrostomía modificada, como la realización del procedimiento bajo fluoroscopia asociado con el uso de agujas más largas en el caso de carcinomatosis peritoneal, o la realización de paracentesis previa cuando exista ascitis $(3,19)$.

Un claro beneficio de la GED sobre el método radiológico es la capacidad para la visualización directa y la evaluación de la cámara gástrica para identificar otra patología, y se encontraron lesiones como esofagitis graves, úlcera gástrica o duodenal activa, gastritis hemorrágica erosiva, o infiltración neoplásica de la pared gástrica reportadas en la literatura, desde el $10 \%$ hasta el $59 \%$ (23).

\section{Desenlaces}

El principal impacto de la GED está dado por la mejoría en la calidad de vida, ya que la resolución y la supervivencia no han mostrado resultados significativos. Existen estudios que han evaluado la GED, dentro de los cuales se describe una cohorte retrospectiva, en la que se evaluaron 439 pacientes con OGIM por condiciones oncológicas gastrointestinales, en quienes se realizó la GED y se observó que la mediana de supervivencia fue de 37 días (24). En otra cohorte retrospectiva con 75 participantes con OGIM a quienes se les realizó GED disminuyó significativamente la frecuencia de emesis diaria y de náuseas, y en el $69 \%$ se presentaron complicaciones menores y en el $24 \%$, complicaciones mayores; las más frecuentes fueron la fuga estromal, dolor leve y obstrucción de la sonda (25).

En la Tabla 2 se resumen 3 estudios en los cuales se específica la neoplasia primaria, porcentaje de éxito técnico, porcentaje de éxito clínico y resolución de la obstrucción, en la que se describe el éxito con el manejo de GED para el manejo de la OGIM. 
En el estudio realizado por Pothuri y colaboradores (26), 94 pacientes con cáncer de ovario requirieron GED para OGIM; la edad media fue 56 años. La media del intervalo desde el diagnóstico inicial de cáncer y la GED fue 3,1 años. El $89 \%$ de los pacientes recibió 3 o más regímenes de quimioterapia antes del procedimiento. 22 de 77 pacientes a quienes se les realizó TAC antes del procedimiento tuvieron un tumor encapsulado del estómago; de estos pacientes, 59 (63\%) tenían ascitis y 25 fueron llevados a paracentesis pre-GED. El $100 \%$ de los pacientes tuvo éxito en la colocación de la sonda y $91 \%$ tuvo mejoría clínica. La media del número de días para obtener mejoría fue 1,7 y todos los pacientes tuvieron mejoría después de 7 días.

Uno de los interrogantes ha sido el manejo de los pacientes con OGIM que cursan con ascitis, ya que en este grupo de pacientes se incrementa el riesgo de complicaciones; sin embargo, se ha demostrado éxito técnico en pacientes con OGIM y ascitis. En el estudio realizado por Shaw y colaboradores (28), en el que se realizó una GED para el manejo de OGIM en 93 pacientes, 13 fueron llevados a paracentesis, 78 pacientes fueron manejados con un catéter intraperitoneal y 2 pacientes no requirieron drenaje; y se presentaron complicaciones en el 13,9\%. Por lo anterior, se indicó el manejo con paracentesis evacuatoria previa al procedimiento y se puso un catéter de drenaje que mejoró la ascitis y favoreció la realización de GED, sin incrementar el riesgo de infección.

En otro estudio prospectivo, en el que se evaluaron 25 pacientes con cáncer ginecológico y gastrointestinal avanzado con OGIM llevados a GED, se describió que existe mejoría significativa en calidad de vida en 16 (64\%) pacientes, empeoramiento no significativo en 7 (28\%), principalmente por la persistencia de síntomas físicos, y 2 (8\%) sin cambios en el puntaje de calidad de vida (29).

\section{Complicaciones}

Existen posibles complicaciones tanto mayores como menores que varían entre el 5,9\% y el 7,8\%, respectivamente. Dentro de las menores se ha descrito el sangrado superficial, infección estromal pericatéter, granulación excesiva del tejido cercano al estroma, obstrucción del catéter, dislocación del catéter y fuga del catéter. Otras complicaciones más graves, pero menos frecuentes son la hemorragia, perforación intestinal, peritonitis, formación de abscesos e infección de tejidos profundos de la piel (3).

Ryan y colaboradores (27) reportaron complicaciones mayores en el 6,7 \% con un paciente que requirió laparotomía emergente 18 días posprocedimiento, luego de que la TAC demostrara que la sonda se encontraba intraperitoneal con necrosis de la pared anterior del estómago y la pared abdominal. Este paciente falleció a las 7 semanas secundario a disfunción multiorgánica. En el estudio de Pothuri y colaboradores (26), las complicaciones asociadas con la GED se presentaron en 17 (18\%) de los pacientes, y la complicación más frecuente fue la fístula en 8 de 94 pacientes $(9 \%)$.

Dado lo anteriormente descrito, se presenta un algoritmo de manejo de la OGIM en la Figura 1.

\section{CONCLUSIONES}

La GED podría ser parte las herramientas de manejo en pacientes seleccionados que cursan con obstrucción intestinal maligna, debido a que apoya la paliación de síntomas, facilita el retorno al sitio del cuidado y podría permitir la posibilidad de reiniciar la vía oral, lo que puede generar cambios significativos en aspectos emocionales, de dignidad y autonomía; así como percepción de bienestar. El impacto sobre la calidad de vida ya comprometida de estos pacien-

Tabla 2. Eficacia de la GED en OGIM secundario a diferentes neoplasias

\begin{tabular}{|c|c|c|c|c|c|}
\hline Estudio & $\begin{array}{l}\text { Pacientes } \\
\text { n (neoplasia } \\
\text { primaria) }\end{array}$ & Éxito técnico & $\begin{array}{l}\text { Éxito } \\
\text { clínico }\end{array}$ & Tolerancia a la dieta & Resolución \\
\hline $\begin{array}{l}\text { Pothuri y } \\
\text { colaboradores (26) }\end{array}$ & 94 (ovario) & $\begin{array}{l}94 \text { total }(100 \%) ; \\
92 \text { GED, } 2 \text { Rx }\end{array}$ & $86(91 \%)$ & $\begin{array}{l}\text { 3: ninguna, 9: sorbos, } 40 \text { : líquidos, } \\
\text { 40: comida blanda/regular, } 2 \text { : } \\
\text { desconocido; } 27 / 34(84,4 \%)\end{array}$ & $\begin{array}{l}\text { 29/94 (31\%) recibieron quimioterapia; } \\
4 / 94(4,3 \%) \text { resolvieron después de } \\
\text { tratamiento oncológico }\end{array}$ \\
\hline $\begin{array}{l}\text { Ryan y } \\
\text { colaboradores (27) }\end{array}$ & 45 (ovario) & $44 / 95(97,8 \%) \mathrm{Rx}$ & - & - & - \\
\hline $\begin{array}{l}\text { Campagnutta y } \\
\text { colaboradores (23) }\end{array}$ & $\begin{array}{l}34 \text { ( } 29 \text { ovario, } 4 \\
\text { útero, } 1 \text { cérvix) }\end{array}$ & $\begin{array}{l}32 / 34 ; 28 / 34 \text { GED, } \\
4 \text { ultrasonografía }\end{array}$ & $\begin{array}{l}27 / 34 \\
(84,4 \%)\end{array}$ & $27 / 34(84,4 \%)$ & $\begin{array}{l}8 / 37(23,5 \%) \text { recibieron quimioterapia; } \\
3 / 34(8,8) \text { resolvieron después de } \\
\text { tratamiento oncológico }\end{array}$ \\
\hline
\end{tabular}

$\mathrm{Rx}$ : radiografía. 
tes de la OGIM y su tratamiento actual con la SNG pueden paliarse con GED; la evidencia disponible de series de casos retrospectiva sugiere que puede ser una alternativa eficaz y relativamente segura en el abordaje de esta seria enfermedad.

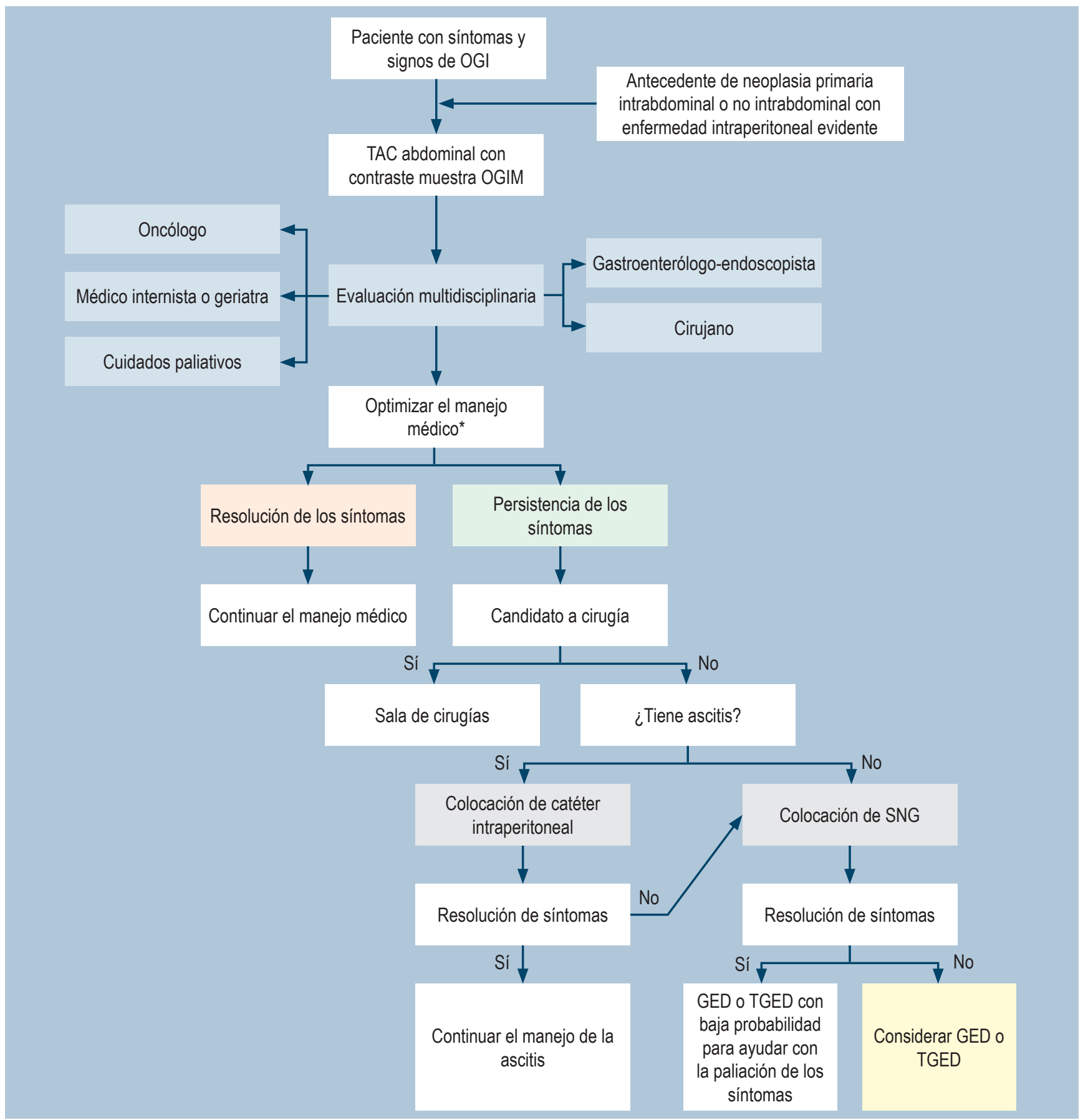

Figura 1. Algoritmo de manejo de OGIM (28). * Optimizar el manejo médico: inhibidores de la bomba de protones (IBP), antihistamínicos bloqueantes $\mathrm{H}_{2}$, anticolinérgicos, corticoides. Octreotida. OGI: obstrucción gastrointestinal. OGIM: obstrucción gastrointestinal maligna; TGED: tubo de gastrostomía endoscópica descompresiva. Adaptado de: Shaw C et al. Ann Surg Oncol. 2013;20(2):497-505. 


\section{REFERENCIAS}

1. Lilley EJ, Scott JW, Goldberg JE, Cauley CE, Temel JS, Epstein AS, et al. Survival, Healthcare Utilization, and End-of-life Care Among Older Adults With Malignancyassociated Bowel Obstruction: Comparative Study of Surgery, Venting Gastrostomy, or Medical Management. Ann Surg. 2018;267(4):692-699.

https://doi.org/10.1097/SLA.0000000000002164

2. Laval G, Marcelin-Benazech B, Guirimand F, Chauvenet L, Copel L, Durand A, et al. Recommendations for bowel obstruction with peritoneal carcinomatosis. J Pain Symptom Manage. 2014;48(1):75-91.

https://doi.org/10.1016/j.jpainsymman.2013.08.022

3. Miller ZA, Mohan P, Tartaglione R, Narayanan G.

Bowel Obstruction: Decompressive Gastrostomies and Cecostomies. Semin Intervent Radiol. 2017;34(4):349-360. https://doi.org/10.1055/s-0037-1608706

4. Jacquet P, Jelinek JS, Steves MA, Sugarbaker PH. Evaluation of computed tomography in patients with peritoneal carcinomatosis. Cancer. 1993;72(5):1631-6.

https://doi.org/10.1002/1097-

0142(19930901)72:5<1631::aid-

cncr2820720523>3.0.co;2-i

5. Berger J, Lester P, Rodrigues L. Medical Therapy of Malignant Bowel Obstruction With Octreotide, Dexamethasone, and Metoclopramide. Am J Hosp Palliat Care. 2016;33(4):407-10. https://doi.org/10.1177/1049909115569047

6. Pujara D, Chiang YJ, Cormier JN, Bruera E, Badgwell B. Selective Approach for Patients with Advanced Malignancy and Gastrointestinal Obstruction. J Am Coll Surg. 2017;225(1):53-59. https://doi.org/10.1016/j.jamcollsurg.2017.04.033

7. Krouse RS. Malignant bowel obstruction. J Surg Oncol. 2019;120(1):74-77. https://doi.org/10.1002/jso.25451

8. Tuca A, Guell E, Martinez-Losada E, Codorniu N. Malignant bowel obstruction in advanced cancer patients: epidemiology, management, and factors influencing spontaneous resolution. Cancer Manag Res. 2012;4:159-69. https://doi.org/10.2147/CMAR.S29297

9. Henry JC, Pouly S, Sullivan R, Sharif S, Klemanski D, Abdel-Misih S, et al. A scoring system for the prognosis and treatment of malignant bowel obstruction. Surgery. 2012;152(4):747-56; discussion 756-7. https://doi.org/10.1016/j.surg.2012.07.009

10. Mirensky TL, Schuster KM, Ali UA, Reddy V, Schwartz PE, Longo WE. Outcomes of small bowel obstruction in patients with previous gynecologic malignancies. Am J Surg. 2012;203(4):472-9. https://doi.org/10.1016/j.amjsurg.2011.07.013

11. Chakraborty A, Selby D, Gardiner K, Myers J, Moravan V, Wright F. Malignant bowel obstruction: natural history of a heterogeneous patient population followed prospectively over two years. J Pain Symptom Manage. 2011;41(2):412-20. https://doi.org/10.1016/j.jpainsymman.2010.05.007

12. Stellato TA, Gauderer MW. Percutaneous endoscopic gastrostomy for gastrointestinal decompression. Ann Surg. 1987;205(2):119-22. https://doi.org/10.1097/00000658-198702000-00002

13. Anthony T, Baron T, Mercadante S, Green S, Chi D, Cunningham J, et al. Report of the clinical protocol committee: development of randomized trials for malignant bowel obstruction. J Pain Symptom Manage. 2007;34(1 Suppl):S49-59. https://doi.org/10.1016/j.jpainsymman.2007.04.011

14. Mudumbi SK, Leonard EV, Swetz KM. Challenges and successes in non-operative management of high-grade malignant bowel obstruction. Ann Palliat Med. 2017;6(Suppl 1): S95-S98. https://doi.org/10.21037/apm.2017.03.07

15. Koo D, Goring T, Pinard K-A, Egan B. Malignant bowel obstruction in advanced cancer. Hosp Med Clin. 2016;5(3):413-24. https://doi.org/10.1016/j.ehmc.2016.02.005

16. Feuer DJ, Broadley KE. Corticosteroids for the resolution of malignant bowel obstruction in advanced gynaecological and gastrointestinal cancer. Cochrane Database Syst Rev. 2000;2000(2):CD001219. https://doi.org/10.1002/14651858.CD001219

17. Suárez Pérez A. Alimentación del paciente de cáncer en fase avanzada y terminal: consideraciones éticas y recomendaciones prácticas. Humanidad Med. 2006;6(2):1-9.

18. Scheidbach H, Horbach T, Groitl H, Hohenberger W. Percutaneous endoscopic gastrostomy/jejunostomy (PEG/PEJ) for decompression in the upper gastrointestinal tract. Initial experience with palliative treatment of gastrointestinal obstruction in terminally ill patients with advanced carcinomas. Surg Endosc. 1999;13(11):1103-5. https://doi.org/10.1007/s004649901182

19. Itkin M, DeLegge MH, Fang JC, McClave SA, Kundu S, d'Othee BJ, et al. Multidisciplinary practical guidelines for gastrointestinal access for enteral nutrition and decompression from the Society of Interventional Radiology and American Gastroenterological Association (AGA) Institute, with endorsement by Canadian Interventional Radiological Association (CIRA) and Cardiovascular and Interventional Radiological Society of Europe (CIRSE). Gastroenterology. 2011;141(2):742-65. https://doi.org/10.1053/j.gastro.2011.06.001

20. Levin DC, Matteucci T. "Turf battles" over imaging and interventional procedures in community hospitals: survey results. Radiology. 1990;176(2):321-4. https://doi.org/10.1148/radiology.176.2.2367646

21. Silas AM, Pearce LF, Lestina LS, Grove MR, Tosteson A, Manganiello WD, et al. Percutaneous radiologic gastrostomy versus percutaneous endoscopic gastrostomy: a comparison of indications, complications and outcomes in 370 
patients. Eur J Radiol. 2005;56(1):84-90.

https://doi.org/10.1016/j.ejrad.2005.02.007

22. Hoffer EK, Cosgrove JM, Levin DQ Herskowitz MM, Sclafani SJ. Radiologic gastrojejunostomy and percutaneous endoscopic gastrostomy: a prospective, randomized comparison. J Vasc Interv Radiol. 1999; 10(4):413-20. https://doi.org/10.1016/s1051-0443(99)70058-8

23. Campagnutta E, Cannizzaro R. Percutaneous endoscopic gastrostomy (PEG) in palliative treatment of non-operable intestinal obstruction due to gynecologic cancer: a review. Eur J Gynaecol Oncol. 2000;21(4):397-402.

24. Pinard KA, Goring TN, Egan BC, Koo DJ. Drainage Percutaneous Endoscopic Gastrostomy for Malignant Bowel Obstruction in Gastrointestinal Cancers: Prognosis and Implications for Timing of Palliative Intervention. J Palliat Med. 2017;20(7):774-778. https://doi.org/10.1089/jpm.2016.0465

25. Dittrich A, Schubert B, Kramer M, Lenz F, Kast K, Schuler $\mathrm{U}$, et al. Benefits and risks of a percutaneous endoscopic gastrostomy (PEG) for decompression in patients with malignant gastrointestinal obstruction. Support Care Cancer. 2017;25(9):2849-2856.

https://doi.org/10.1007/s00520-017-3700-1
26. Pothuri B, Montemarano M, Gerardi M, Shike M, BenPorat L, Sabbatini P, et al. Percutaneous endoscopic gastrostomy tube placement in patients with malignant bowel obstruction due to ovarian carcinoma. Gynecol Oncol. 2005;96(2):330-4. https://doi.org/10.1016/j.ygyno.2004.09.058

27. Ryan JM, Hahn PF, Mueller PR. Performing radiologic gastrostomy or gastrojejunostomy in patients with malignant ascites. AJR Am J Roentgenol. 1998;171(4):1003-6. https://doi.org/10.2214/ajr.171.4.9762985

28. Shaw C, Bassett RL, Fox PS, Schmeler KM, Overman MJ, Wallace MJ, et al. Palliative venting gastrostomy in patients with malignant bowel obstruction and ascites. Ann Surg Oncol. 2013;20(2):497-505. https://doi.org/10.1245/s10434-012-2643-5

29. Zucchi E, Fornasarig M, Martella L, Maiero S, Lucia E, Borsatti E, et al. Decompressive percutaneous endoscopic gastrostomy in advanced cancer patients with small-bowel obstruction is feasible and effective: a large prospective study. Support Care Cancer. 2016;24(7):2877-82. https://doi.org/10.1007/s00520-016-3102-9 